\title{
EFECTO DEL CLIMA, DE LAS CARACTERÍSTICAS DE LA HOJA Y DE LA METODOLOGÍA DE MEDICIÓN EN EL POTENCIAL HÍDRICO XILEMÁTICO EN PALTO (Persea americana Mill.)
}

\author{
Effects of environment, leaf characteristics and measuring methodology on \\ stem water potential in avocado trees (Persea americana Mill.)
}

\author{
Raúl Ferreyra E. ${ }^{1 *}$, Gabriel Selles V. ${ }^{1}$, Patricio Maldonado B. ${ }^{2}$, José Celedón de A. ${ }^{2}$ \\ y Pilar Gil M. ${ }^{2}$
}

A B S T R A C T

A research was developed during 2003 - 2004 at an avocado (Persea americana Mill.) orchard cv. Hass grafted on Mexicola rootstock. The orchard was located in the $\mathrm{V}^{\text {th }}$ Region of Chile ( $32^{\circ} 53^{\prime}$ lat. S; $71^{\circ} 16^{\prime}$ long. W). The orchard was under optimal water and nutritional condition. Several experiences were carried out to optimize midday stem water potential ( $\left.\Psi_{\mathrm{xm} i n}\right)$ measurements, effected by means of pressure chamber techniques: effect of vapor pressure deficit (DVP), leaf age, time of equilibrium of leaf and stem water potential after leaf envelopment, minimum time between leaf detachment and stem water potential measurement in the pressure chamber, leaf location on the tree and the effect of high humidity conditions on detached leaves prior to measurements. The results showed that in avocado trees $\Psi$ xmin were stable between 12:30 and 17:30 h. The less variability of measurements was obtained using 10 to 12 months old leaves, under exposure to sunlight. The pressure chamber measurements should be carried out $15 \mathrm{~min}$ after the leaf was enveloped. $\Psi_{\mathrm{x}}$ should be measured no longer than $1 \mathrm{~min}$ after the leaf is detached from the tree; however, this time could be longer if the leaves are kept in high humidity conditions before measurements.

Key words: xylem water potential, aguacate, plant water status, Persea americana Mill.

\author{
R E S U M E N
}

Se realizó una investigación durante la temporada 2003/2004 en un huerto de paltos (Persea americana Mill.) cv. Hass, sobre portainjerto Mexicola, con el objeto de mejorar la metodología de medición del potencial hídrico xilemático medido a medio día ( $\Psi \mathrm{xm})$, utilizando la técnica de la cámara de presión. El huerto estaba localizado en la V Región ( $32^{\circ} 53^{\prime}$ lat. Sur; $71^{\circ} 16^{\prime}$ long. Oeste). Los árboles se encontraban en etapa productiva y sin restricciones hídricas ni nutricionales. Se realizaron varios experimentos con el objetivo de optimizar y simplificar las mediciones del potencial hídrico xilemático a mediodía ( $\Psi \times \mathrm{xmin})$ : efecto del déficit de presión de vapor del aire (DVP), de la edad de la hoja, del tiempo de equilibrio al cubrir la hoja, del tiempo transcurrido entre corte de la hoja y medición de potencial, de la ubicación de la hoja en el árbol, y de mantención de la hoja en condiciones de alta humedad relativa después de ser cortada del árbol. Los resultados obtenidos muestran, entre otros antecedentes, que el $\Psi$ xmin en el palto presenta valores estables entre las 12:30 y 17:30 h. Las hojas que presentan la menor variabilidad son las de 10 a 12 meses de edad, expuestas al sol. El tiempo mínimo de equilibrio para medir el $\Psi$ xmin es de 15 min luego de envuelta la hoja. Una vez desprendida la hoja del árbol, la medición se debe realizar en un lapso de tiempo no superior a $1 \mathrm{~min}$, sin embargo, se podría aumentar el tiempo entre el corte de la hoja y la medición del potencial hídrico si éstas se mantienen en condiciones de alta humedad relativa.

Palabras clave: potencial hídrico xilemático, aguacate, estado hídrico de la planta, Persea americana Mill.

\footnotetext{
${ }^{1}$ Instituto de Investigaciones Agropecuarias, Centro Regional de Investigación La Platina, Santa Rosa 11610, Santiago, Chile.

E-mail: rferreyr@inia.cl*Autor para correspondencia.

${ }^{2}$ Instituto de Investigaciones Agropecuarias, Centro Regional de Investigación La Cruz, Chorrillos 86, La Cruz, Chile. Proyecto financiado por FDI-CORFO.

Recibido: 28 de diciembre de 2005. Aceptado: 8 de mayo de 2006.
} 


\section{INTRODUCCIÓN}

A nivel mundial, los rendimientos promedio de los huertos de paltos, o aguacate (Persea americana Mill.) son bajos en comparación con otras frutas de pulpa. Esto se debe en gran parte a que para producir frutos de semilla grande y ricos en aceite se requiere un alto costo en fotosintatos (Wolstenholme, 1986). En Chile hay huertos que mantienen producciones estables de alrededor de $25 \mathrm{t} \mathrm{ha}^{-1}$, sin embargo, el rendimiento promedio de los huertos adultos está alrededor de las $9 \mathrm{t} \mathrm{ha}^{-1}$, debido principalmente a problemas de asfixia radicular, ya que el palto es muy sensible a la falta de aire en el suelo, y la mayoría de las plantaciones en el país se han realizado en terrenos con baja capacidad de aire, cercanas al 15\%, y alta densidad aparente, 1,3 a 1,4 $\mathrm{g} \mathrm{cm}^{-3}$ (Nagera, 2006).

El palto es muy sensible a la falta de aire en el suelo, debido a que es originario de zonas donde predominan los suelos Andisoles, derivados de cenizas volcánicas (Aguilera y Salazar, 1991), los cuales se caracterizan por presentar alta capacidad de aire, cercana al 46\%, baja densidad aparente, 0,5$0,8 \mathrm{~g} \mathrm{~cm}^{-3}$, y pH ácido, entre 5 y 6 . Ferreyra et al. (2006) encontraron que el palto cv. Hass, sobre portainjerto Mexicola se desarrolló mejor en suelos arenosos, de alta capacidad de aire, que en suelos franco arcillosos o limosos que presentaron tasas de difusión de oxígeno inferiores a $0,17 \mu \mathrm{g} \mathrm{cm}^{-2} \mathrm{~min}^{-1}$.

Por lo indicado anteriormente, uno de los principales factores que afectan la producción y calidad de la fruta, sobre todo en condiciones de suelo desfavorables para el desarrollo del palto, es una inadecuada relación entre el agua y aire en la zona de la rizósfera, producto del manejo del riego. Un exceso o falta de agua durante el crecimiento del palto limita la producción y la calidad de la fruta, particularmente si el estrés se produce entre primavera e inicio de verano (Whiley et al., 1988a; 1988b).

Entre las soluciones para superar este problema destacan la búsqueda de portainjertos resistentes a baja aireación en el suelo. No obstante, para huertos ya establecidos deben buscarse nuevas alternativas de manejo que permitan disminuir la incidencia de este inconveniente. Una forma de lograr esto es mantener en los suelos contenidos de humedad inferiores a capacidad de campo, lo que permitiría una mejor relación aire:agua en el suelo. Sin em- bargo, esto obliga a un adecuado control del riego de manera de evitar períodos de déficit hídrico.

El riego se puede controlar definiendo el momento oportuno de aplicación de agua, a través de mediciones del estado hídrico de las plantas, como es el potencial hídrico de la hoja $(\Psi$ h) (Boyer, 1995). Sin embargo, su valor como indicador del estado hídrico del cultivo ha sido cuestionado por su variabilidad, dada la influencia de factores microambientales, especialmente la exposición a la luz solar directa que presentan las hojas a medir (Shackel et al, 1997). Debido a lo anterior, algunos autores miden el potencial hídrico temprano en la mañana, antes de la salida del sol (Williams y Araujo, 2002). Sin embargo, esta medición es poco sensible a la disponibilidad de agua en el suelo, ya que el potencial hídrico de la planta tiende a equilibrarse con las zonas más húmedas del suelo (Garnier y Berger, 1987, Ameglio et al., 1999). Por otra parte, desde el punto de vista práctico, es difícil que el $\Psi \mathrm{h}$ medido en la madrugada pueda ser utilizado como controlador rutinario del riego, debido a la hora (antes de la salida del sol) en que debe realizarse la medición en el campo.

Es posible eliminar los factores microambientales que afectan las mediciones del $\Psi \mathrm{h}$ indicados anteriormente, al bloquear la transpiración de la hoja, antes de ser cortada de la planta (Garnier y Berger, 1985; Olien y Lakso, 1986; Shackel et al., 1997). La metodología para lograr lo anterior consiste en, previo a la medición, poner las hojas que se van a medir en una bolsa plástica, y luego envolverlas en papel aluminio, para impedir el paso de la luz, bloqueando de esta forma la transpiración de la hoja. En estas condiciones el potencial hídrico medido representa el potencial promedio de la ramilla que sostiene la hoja. Previo a la medición, las hojas deben permanecer envueltas por un período de $2 \mathrm{~h}$, para que $\Psi$ h se equilibre con el potencial hídrico xilemático o del brote $(\Psi \mathrm{x})$ (McCutchan y Shackel, 1992). Sin embargo, Fulton et al. (2001) indican que en nogales (Junglans regia L.), almendros (Prunus dulcis (Mill.) y ciruelos (Prunus domesti$c a$ L.) este equilibrio se logra después de 10 min de colocada la hoja en una bolsa cuyo interior es de plástico y exterior de papel aluminio. En palto no existe información al respecto.

De esta forma, el $\Psi \mathrm{x}$ es menos influenciado por cambios microambientales de corto plazo que afec- 
tan la transpiración de la hoja y por lo tanto al $\Psi \mathrm{h}$. En consecuencia, el $\Psi \mathrm{x}$ es una medida más apropiada del estado de hídrico de la planta (McCutchan y Shackel, 1992). Diferentes estudios han demostrado que el $\Psi_{\mathrm{x}}$ medido con cámara de presión es un buen indicador del estrés hídrico en árboles frutales. Naor et al. (1995) encontró una buena correlación entre $\Psi \mathrm{x}$ y el rendimiento y calibre en manzanos (Pyrus malus L.). Datos similares encontraron Ferreyra et al. (2002) y Ferreyra et al. (2004) en duraznero (Prunus persica (L.) Batsch) y vides viníferas (Vitis vinifera L.). Naor (1998) y Ferreyra et al. (2002) comprobaron que la conductancia estomática en nectarines (Prunus persica var nectarina (Aiton) Maxim.) y vid vinífera está altamente correlacionada con $\Psi_{\mathrm{x}}$. El $\Psi_{\mathrm{x}}$ mínimo o de mediodía ( $\left.\Psi_{\mathrm{x} m i n}\right)$, ha sido propuesto por varios autores como un indicador estándar para determinar el estado hídrico de la planta con fines de riego (Fereres y Goldhamer, 1990; Shackel et at., 1997; Naor y Peres, 2001; Ferreyra et al., 2002). Sin embargo, los tiempos necesarios para producir el equilibrio del potencial en la hoja y el escaso tiempo disponible para realizar la medición a mediodía, sobre todo cuando el huerto involucra una gran superficie, dificultan la adopción de esta técnica por parte de los agricultores.

Por lo indicado anteriormente, el objetivo de este estudio fue determinar el efecto del déficit de presión de vapor del aire (DVP) sobre el $\Psi$ xmin, la edad de la hoja en que se realiza la medición, la ubicación de la hoja en el árbol, el tiempo transcurrido entre cubrir la hoja y realizar la medición de $\Psi \mathrm{x}$, el tiempo entre la toma de la muestra y la medición en la cámara de presión en condiciones de terreno, $\mathrm{y}$, finalmente, el tiempo de mantención de la hoja en condiciones de alta humedad relativa, después de ser cortada del árbol, antes de la medición. La respuesta a estas interrogantes permitirá optimizar el uso del $\Psi$ xmin como técnica de control de riego en paltos.

\section{MATERIALES Y MÉTODOS}

Los experimentos se condujeron en la localidad de Quillota (32 $53^{\prime}$ lat. Sur; $71^{\circ} 16^{\prime}$ long. Oeste), Región de Valparaíso, zona central de Chile, durante la temporada 2003-2004. Esta zona es semiárida, sin lluvia de verano, por lo cual el clima corresponde a mediterráneo templado, con temperaturas medias anuales de $15,3^{\circ} \mathrm{C}$, con una máxima media del mes más cálido (enero) de $27^{\circ} \mathrm{C}$ y una mínima me- dia del mes más frío (julio) de $5,5^{\circ} \mathrm{C}$. El período libre de heladas es de 9 meses, de septiembre a mayo, y el régimen hídrico se caracteriza por una precipitación anual de $437 \mathrm{~mm}$, concentrada en los meses de junio a agosto.

Se implementaron siete experimentos en árboles de palto cv. Hass, sobre porta injertos Mexicola, regados con un microaspersor por planta. La edad de los árboles, dependiendo del ensayo, varió entre 4 y 7 años, encontrándose todos en etapa productiva y sin restricciones hídricas ni nutricionales.

A continuación se detallan los experimentos realizados:

Determinación del período durante el día cuando el $\Psi_{\mathbf{x}}$ es estable. Se evaluó la variación diaria del potencial hídrico xilemático, entre las 07:00 y las 19:00 h. Estas mediciones se realizaron en el período de verano (diciembre-enero) en 11 oportunidades. En forma simultánea se evaluó el DVP del aire. Cada determinación de potencial hídrico se realizó en cuatro hojas por árbol.

\section{Determinación del tiempo mínimo de equilibrio entre el $\Psi_{\mathbf{h}}$ y el $\Psi_{\mathbf{x m}} \mathrm{min}$. Se escogieron 28 hojas, las cuales se introdujeron en bolsas cuyo interior era de plástico y exterior de papel aluminio, de ma- nera de bloquear su transpiración. Una vez envuel- tas, las hojas se dejaron en el árbol durante diferen- tes tiempos, los cuales variaron entre 4 y $80 \mathrm{~min}$. Las mediciones se realizaron a mediodía. La prime- ra medición se realizó después de 4 min, para prose- guir las mediciones a intervalos entre 2 y $10 \mathrm{~min}$.}

Efecto de la ubicación de la hoja en el árbol sobre el $\Psi_{x m i n}$. En cada árbol se midió el $\Psi_{x m i n}$ en la parte del follaje expuesto a la radiación solar directa y en aquella parte sombreada. Las mediciones se realizaron en una plantación con las hileras en dirección Norte-Sur, de modo que una sola parte recibiera radiación directa.

Efecto del tiempo transcurrido entre la toma de la muestra y la medición en el $\Psi x$ xmin. En este experimento se midió $\Psi$ xmin a 33 hojas que tenían distinto tiempo entre el corte del árbol y la medición (1 a $27 \mathrm{~min}$ ). El $\Psi_{\mathrm{xmin}}$ de la primera muestra se midió después de 1 min de haberse cortado, para luego proseguir las mediciones en hojas con mayor tiempo entre el corte y la medición. 
Efecto de la edad de la hoja en el $\Psi$ xmin. Se determinó $\Psi$ xmin en hojas de distintas edades: 1 mes, 4 meses y 10 a 12 meses de edad; se utilizaron 10 hojas de cada edad.

Efecto del DVP máximo en el $\Psi x \operatorname{xmin}$. Durante primavera y verano, en 32 oportunidades se midieron el $\Psi$ xmin y DVP del aire; se utilizaron cuatro hojas por determinación.

Efecto de la conservación de la hoja en condiciones de alta humedad relativa sobre el $\Psi_{x m i n}$. En este experimento se midió $\Psi$ xmin en cuatro árboles de palto que se encontraban en distintas condiciones de estrés hídrico. En cada árbol se midió el $\Psi$ xmin a cuatro hojas, de la cuales dos se midieron inmediatamente después de cortadas del árbol, y las restantes después de $1 \mathrm{~h}$ de ser separadas de la planta y mantenidas en una caja de PVC aislada térmicamente y con hielo en el interior, de manera de tener una humedad relativa (HR) cercana a $100 \%$ según mediciones realizadas.

El $\Psi x m i n$ en los distintos ensayos se midió, en días con sol, entre 13:00 y 16:00 h, utilizando una cámara de presión (Soil Moisture Equipment Corp., Modelo CHANGE C, Santa Bárbara, California, USA), de acuerdo a la metodología descrita por (McCutchan y Shackel, 1992). Las hojas medidas se encontraban en brotes de condiciones homogéneas en cuanto a exposición (cara expuesta al sol), edad y altura (1,5 $\mathrm{m}$ del suelo).

El DVP se determinó cada 15 min, durante las mediciones de $\Psi$ xmin, con un psicrómetro ventilado (Fuese, Modelo G-5069, Berlín, Alemania) el cual medía la temperatura del aire y la humedad relativa.

Se realizaron análisis de regresiones usando PROCREG del programa de análisis estadístico SAS (SAS, 1989).

\section{RESULTADOS Y DISCUSIÓN}

Valores de referencia y período durante el día cuando se puede medir en forma estable el $\Psi_{\mathbf{x}}$

Para las condiciones climáticas de Quillota, el $\Psi_{\mathrm{X}}$ en el palto presenta valores muy similares, sin diferencias significativas, entre las 12:30 y 17:30 h (Figura 1). Durante este período los valores diarios medidos fueron los mínimos, fluctuando entre $-0,36$ y -0,40 MPa, con un promedio de $-0,38 \mathrm{MPa}$.

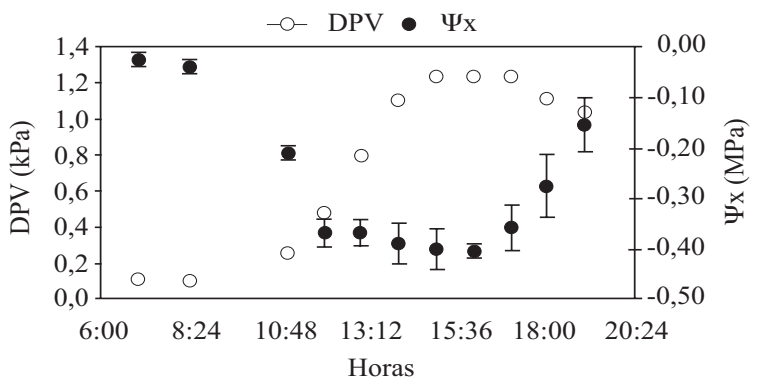

Figura 1. Efecto del déficit de presión de vapor (DVP) durante el día sobre el potencial hídrico xilemático $(\Psi \mathbf{x})$.

Figure 1. Effect of vapor pressure deficit (DPV) during the day on stem water potential $\left(\Psi_{\mathbf{x}}\right)$.

Las barras verticales representan la desviación estándar.

A mediodía, las variaciones en el DPV del aire tienen poco efecto sobre el $\Psi$ xmin (Figura 2). El $\Psi$ xmin fluctúa entre -0,40 y -0,50 MPa (variación del 20\%) para DVP que oscila entre 1,5 y $3,0 \mathrm{kPa}$ respectivamente (variación del 50\%).

De estos resultados se puede inferir que durante el verano se disponen de $5 \mathrm{~h}$ en el día (12:30 a 17:30 h) para medir el $\Psi$ xmin con fines de control del riego, y que en paltos creciendo sin restricción hídrica este valor varía entre $-0,4$ y $-0,5 \mathrm{MPa}$.

Determinación del tiempo mínimo de equilibrio para la determinación del $\Psi_{\mathbf{x}}$

El $\Psi$ h se equilibra con el $\Psi$ x después de 15 min de haberse bloqueado la transpiración al introducir la

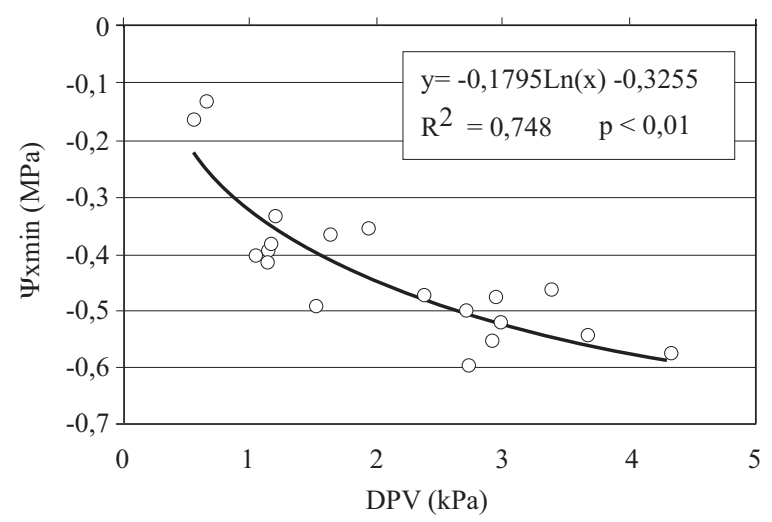

Figura 2. Efecto del déficit de presión de vapor (DVP) sobre el potencial hídrico xilemático a mediodía ( $\Psi_{\mathrm{xmin}}$ ).

Figure 2. Effect of vapor pressure deficit (DVP) on the midday stem water potential $\left(\Psi_{\mathrm{xm}} \mathrm{min}\right)$. 
hoja en bolsas cuyo interior era de plástico y exterior de papel aluminio (Figura 3), lo cual coincide con los datos reportados por Fulton et al. (2001). Esto indicaría que se puede medir el $\Psi \mathrm{x}$ en hojas de paltos después de 15 min de bloqueada la transpiración en las hojas a medir. Además, en la Figura 3 se puede observar que los valores de potencial hídrico medidos recién cubiertas las hojas $(\Psi \mathrm{h})$ presentan una mayor variación $(-0,6$ a $-0,8 \mathrm{MPa})$ que los potenciales evaluados después de $15 \mathrm{~min}(-0,47$ a $-0,55 \mathrm{MPa})$, lo que concuerda con lo reportado por Shackel et al. (1997) que indican que el $\Psi$ h es más variable que el $\Psi_{\mathrm{x}}$.

\section{Efecto de la ubicación de la hoja en el árbol en el $\Psi x$ min}

Las hojas en el árbol expuestas al sol presentaron $\Psi$ xmin más negativos (-0,52 MPa), que las hojas de la cara expuesta a la sombra (-0,40 MPa), aunque se bloqueó la transpiración de éstas antes de ser cortadas de la planta. La desviación estandar (DS) de las mediciones fue baja. Las hojas expuestas al sol presentaron DS de 0,065 MPa y las de la cara expuesta a la sombra DS de $0,018 \mathrm{MPa}$ (datos no mostrados).

\section{Efecto de la edad de la hoja en la variación del $\Psi$ xmin}

Las determinaciones en hojas de 1 mes presentaron $\Psi$ xmin promedio de $-0,55 \mathrm{MPa}$ y una DS de 0,12 $\mathrm{MPa}$; en hojas de 4 meses el $\Psi$ xmin fue de $-0,52$ $\mathrm{MPa}$, con una DS de $0,13 \mathrm{MPa}$, y en hojas de 10 a 12 mes el $\Psi$ xmin fue de -0,49 MPa con una DS de $0,1 \mathrm{MPa}$. Las hojas más jóvenes presentan un potencial 0,06 MPa más bajo que las hojas más adultas y una mayor DS. El $\Psi \mathrm{x}$ presenta menor variación al ser medido en hojas de 10 a 12 meses. Esto

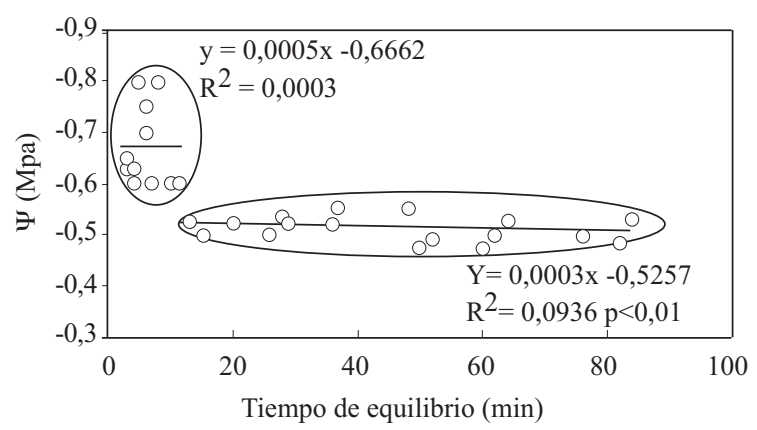

Figura 3. Efecto del tiempo de equilibrio en el potencial hídrico $(\Psi)$ a mediodía bajo condiciones de campo.

Figure 3. Effect of the time of equilibrium on the midday water potential $(\Psi)$ under field conditions. se debería a que las hojas nuevas pueden ser afectadas por la presión que ejerce la goma de la cámara de presión sobre el pecíolo de la hoja, retrasando la aparición de la savia en el xilema. Una variación de 0,06 MPa puede ser importante en el control del riego, según datos reportados por Lampinen (2004), donde indica que el nogal presenta $\Psi_{\mathrm{X}}$ entre $-0,3$ y $-0,5 \mathrm{MPa}$ cuando está sin restricción hídrica, y debiera regarse cuando disminuye este valor en -0,2 ó -0,3 MPa.

Efecto sobre el $\Psi_{x}$ del tiempo transcurrido entre corte de la hoja y medición

El tiempo que transcurre entre el corte de la hoja y la medición es una variable que afecta la determinación del $\Psi_{\mathrm{x}}$ (Figura 4). Si la medición se realiza cuando ha pasado más de 1 min de cortada la hoja, los valores medidos son menores a los reales, producto de la deshidratación de la hoja, y no reflejan el estado hídrico real del brote. Sin embargo, este trabajo muestra datos que permitirían aumentar el tiempo entre el corte de la hoja y la medición del potencial, si las hojas son cortadas e inmediatamente guardadas en una caja de PVC aislada térmicamente con humedad relativa cercana al $100 \%$. El $\Psi \mathrm{x}$ en hojas de un mismo árbol medidas inmediatamente luego de cortadas y otras después de 60 min de almacenamiento en condiciones de oscuridad, con $100 \%$ HR y $5^{\circ} \mathrm{C}$, muestra valores similares (Figura 5). El uso de esta metodología para medir el $\Psi_{\mathrm{x}}$ es factible de implementar, pero requeriría medir un mayor número de hojas por árbol, ya que aumenta la variabilidad. Según la me-

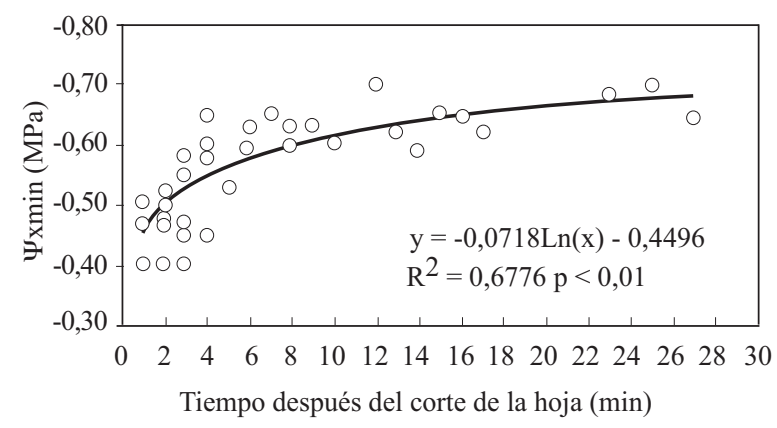

Figura 4. Efecto del tiempo transcurrido entre corte y la medición de la hoja, sobre el potencial hídrico

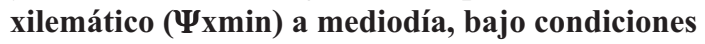
de campo.

Figure 4. Effect of time lapse between leaf cut and measure, on the midday stem water potential ( $\left.\Psi_{\mathrm{Xmin}}\right)$ under field conditions. 


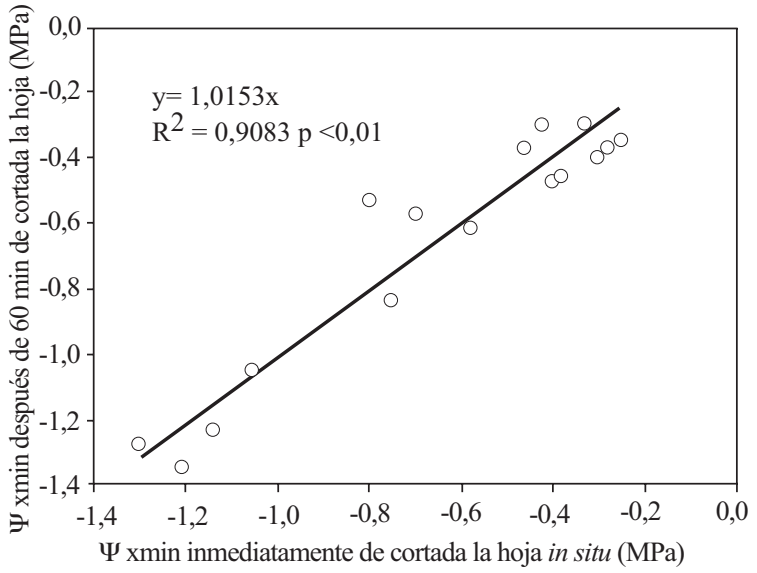

Figura 5. Efecto de la conservación de la hoja en condiciones de alta humedad relativa sobre el potencial hídrico xilemático ( $\Psi_{\mathbf{x} \text { min). }}$.

Figure 5. Effect of keeping the leaf under high relative humidity, on the stem water potential ( $\left.\Psi_{\mathbf{x}} \mathrm{min}\right)$.

todología para datos continuos descrita por Cochran (1971), el tamaño de la muestra debe ser de tres hojas por planta si las mediciones se realizan inmediatamente de cortadas las hojas, y de seis hojas por planta, para evaluaciones después de $60 \mathrm{~min}$ de almacenamiento en condiciones de alta humedad relativa.

\section{CONCLUSIONES}

El presente estudio permite concluir lo siguiente:

- Los valores de $\Psi x$ medidos a mediodía en el palto son estables entre las 12:30 y 17:30 h, lo que permite disponer de $5 \mathrm{~h}$ para realizar mediciones de $\Psi$ xmin.

- Los paltos sin restricción hídrica presentan valores $\Psi$ xmin de entre -0,40 y -0,50 MPa, para valores de DVP de entre 1,4 y 3,0 $\mathrm{kPa}$, respectivamente.

- En las hojas de palto el equilibrio del $\Psi x$ se logra a los 15 min de introducir la hoja en bolsas cuyo interior es de plástico y exterior de papel aluminio.

- En condiciones de campo, el potencial hídrico debe ser medido en un lapso no superior a $1 \mathrm{~min}$ después de cortar la hoja.

- El tiempo que transcurre entre el corte de la hoja y la medición del potencial hídrico en la cámara de presión puede ser aumentado hasta $60 \mathrm{~min}$, al mantener las hojas en una caja de PVC aislada térmicamente y con alta humedad relativa.

- Las hojas que presentan la menor variabilidad son las de 10 a 12 meses de edad, expuestas al sol. Las hojas expuestas al sol presentan valores de $\Psi \mathrm{x}$ más negativos, que las hojas de la cara expuesta a la sombra, aun cuando se haya bloqueado su transpiración.

\section{LITERATURA CITADA}

Aguilera, J., y S. Salazar. 1991. The avocado industry in Michoacan, México. South African Avocado Growers' Association Yearbook No 14. p. 94-97.

Ameglio, T., P. Archer, M. Cohen, C. Valancogne, F. Daudet, and P. Cruiziat. 1999. Significance and limits in the use of predawn leaf water potential for tree irrigation. Plant Soil 207:155-167.

Boyer, J.S. 1995. Measuring the water status of plants and soils. 178 p. Academic Press, San Diego, California, USA.

Cochran, W.G. 1971. Técnica de muestreo. 507 p. Compañía Ed. Continental S.A., México.

Fereres, E., and D. Goldhamer. 1990. Deciduous fruit and nut trees. Monograph $\mathrm{N}^{\circ}$ 30. p. 987-1017. In Steward, B.A., and Nielsen, D.R. (eds.). Irrigation of agricultural crops. American Society of Agronomy, Madison, Wisconsin, USA.
Ferreyra, R., V. Selles, y G. Lemus. 2002. Efecto del estrés hídrico durante la fase II del crecimiento del fruto del duraznero cv. Kakamas en el rendimiento y estado hídrico de las plantas. Agric. Téc. (Chile) 62:565-573.

Ferreyra, R., V. Selles, P. Maldonado, J. Celedón, y A. Torres. 2006. Efecto de la macroporosidad y atmósfera del suelo en el estado hídrico del palto. 208 p. V Congreso Internacional de Ingeniería Agrícola. 9 - 12 mayo. Universidad de Concepción, Facultad de Ingeniería Agrícola - Instituto de Investigaciones Agropecuarias, Centro Regional de Investigación Quilamapu, Chillán, Chile.

Ferreyra, R., V. Selles, J. Peralta, and J. Valenzuela. 2004. Effect of water stress applied at different development periods of Cabernet Sauvignon grapevine on production and wine quality. Acta Hortic. 646:27-33. 
Fulton, A., R. Buchner, B. Olson, L. Schwankl, C. Gilles, N. Betagna et al. 2001. Rapid equilibration of leaf and stem water potential under field conditions in almonds, walnuts, and prunes. HortTechnology 11:609-615.

Garnier, E., and A. Berger. 1985. Testing water potential in peach trees as an indicator of water stress. J. Hortic. Sci. 60:47-56.

Garnier, E., and A. Berger. 1987. The influence of drought on stomatal conductance and water potential on peach trees growing in the field. Sci. Hortic. 32:249-263.

Lampinen, B.D. 2004. Walnut production and orchard management in California. 9 p. Seminario Internacional El Nogal y otros Frutos de Nuez. 17 al 19 agosto 2004. Santiago. Instituto de Investigaciones Agropecuarias, Santiago, Chile.

McCutchan, H., and K.A. Shackel. 1992. SWP as a sensitive indicator of water stress in prune trees (Prunus domestica L. cv. French). J. Am. Soc. Hortic. Sci. 117:607-611.

Nagera, F. 2006 Caracterización de suelos alcalinos cultivados con paltos (Persea americana Mill.) en la V Región de Chile y aplicación de ácido sulfúrico como corrector de pH. 41 p. Tesis Ingeniero Agrónomo. Universidad de Chile, Facultad de Ciencias Agronómicas, Santiago, Chile.

Naor, A. 1998. Relationships between leaf and stem water potential and stomatal conductance in three fieldgrown woody species. J. Hortic. Sci. 73:431-436.

Naor, A., I. Klein, and I. Doron. 1995. Stem water potential and apple fruit size. J. Am. Soc. Hortic. Sci. 120:577- 582.
Naor, A., and M. Peres. 2001. Pressure increase rate affects the accuracy of stem water potential measurements in deciduous trees using the pressure chamber technique. J. Hortic. Sci. Biotech. 76:661-663.

Olien, W.C., and A.N. Lakso. 1986. Effect of rootstock on apple (Malus domestica) tree water relations. Physiol. Plant. 67:421-430.

SAS Institute. 1989. SAS User's guide. Versión 8. SAS Institute Inc., Cary, North Carolina, USA.

Shackel, K.A., H. Ahmadi, W. Biasi, R. Buchner, D. Goldhamer, S. Gurusinghe et al. 1997. Plant water status as an index of irrigation need in deciduous fruti trees. HortTechnology 7:23-29.

Whiley, A.W., K.R. Chapman, and J.B. Saranah. 1988a. Water loss by floral structures of avocado (Persea americana Mill.) cv. Fuerte during flowering. Aust. J. Agric. Res. 39:457-467.

Whiley, A.W., J.B. Saranah, B.W. Cull, and K.G. Pegg. 1988b. Manage avocado tree growth cycles for productivity gains. Queensland Agric. J. 114:29-36.

Williams, L.E., and F.J. Araujo. 2002. Correlation among predawn leaf, and midday stem water potential and their correlations with other measures of soil plant water status in Vitis vinifera. J. Am. Soc. Hortic. Sci. 127:448-454.

Wolstenholme, B. 1986. Energy costs of fruiting as a yield-limiting factor with special reference to avocado. Acta Hortic. 175:121-126. 